\title{
Quand les cristaux font la lumière sur la structure des canaux ioniques
}

$\mathbf{L}$ es canaux ioniques sont des constituants des membranes cellulaires permettant les échanges transmembranaires d'ions selon leur gradient électrochimique. Ils constituent ainsi des éléments fondamentaux de la signalisation cellulaire, impliqués dans des phénomènes aussi divers que la conduction et la transmission de l'influx nerveux, la contraction musculaire, la libération et la réponse hormonales, les transports épithéliaux et la transduction des signaux mécaniques. Selon le stimulus qui contrôle l'ouverture ou la fermeture du canal, on distingue les canaux dépendant du potentiel, de la fixation d'un ligand, ou de la tension membranaire.

De nombreuses données électrophysiologiques, biochimiques et génétiques ont été accumulées sur les canaux ioniques, mais leur structure tridimensionnelle restait jusqu'ici inconnue. En effet, les protéines membranaires sont difficiles à cristalliser et la détermination de leur structure par diffraction aux rayons $\mathrm{X}$ est peu avancée: à ce jour une dizaine de structures est connue contre plusieurs milliers dans le cas des protéines solubles. La détermination de la structure des canaux ioniques est pourtant une étape essentielle dans la compréhension de leur fonctionnement et des modifications engendrées par les mutations des gènes qui les codent, mutations responsables de nombreuses maladies génétiques. Un pas important vient d'être franchi avec la récente cristallisation et la résolution de la structure de deux canaux ioniques de la membrane plasmique bactérienne: le canal $\mathrm{K}^{+}$de Streptomyces lividans (KcsA) [1, 2] et le canal mécanosensible de Mycobacterium tuberculosis (MscL) [3]. L'information obtenue sur les canaux bactéricns définit des bases structurales qui seront vraisemblablement applicables aux canaux eucaryotes et constitue par là même une avancée majeure de la biologie cellulaire et moléculaire. Nous proposons ici un bref résumé des dernières données concernant la structure tridimensionnelle de ces canaux ioniques. Ce thème a fait l'objet d'un symposium lors du $10^{\mathrm{e}}$ Colloque canaux ioniques (LaLonde-les-Maures, septembre 1999). Nous comparons ces résultats récents avec ceux qui ont été obtenus par l'analyse en microscopie électronique de cristaux bidimensionnels du récepteur nicotinique de l'acétylcholine (nAChR) d'un sélacien, la Torpille, dont la dernière résolution, loin d'approcher celle des études de diffraction aux rayons $X$, atteint néanmoins 4,6 ̊ [4,5].

KcsA fait partie du groupe le plus diversifié des canaux ioniques, celui des canaux $\mathrm{K}^{+}$. KcsA est un canal homo-tétramérique, c'est-à-dire formé par l'association de quatre sous-unités $\alpha$ identiques, qui comprennent chacune deux segments transmembranaires (STM) hélicoïdaux et un domaine $P$ (séquence constituant le pore; figure 1). Cette sous-unité de 160 acides aminés présente une forte homologie avec la région du pore des canaux potassiques eucaryotes. L'activation du canal KcsA, dont nous ignorons encore la fonction, dépend d'un abaissement du pH [6].

Le clonage du MscL a constitué la première identification moléculaire d'un canal mécanosensible [7]. Il s'agit d'un homopentamère constitué d'une petite sous-unité de 136 acides aminés et comprenant deux STM (figure 2) [3]. Une augmentation de la tension membranaire entraîne l'ouverture du MscL qui forme un pore non sélectif de très grande conductance, impliqué dans la protection des bactéries contre le choc osmotique $[8,9]$.
Le nAChR est un canal cationique activé par un ligand, qui assure la transmission du signal entre cellules excitables. L'acétylcholine (ACh) libérée dans l'espace synaptique se lie sur le $\mathrm{nAChR}$ et provoque l'ouverture du canal. Il s'agit d'un complexe glycoprotéique formé de cinq sousunités homologues comprenant quatre STM, organisées selon un axe de pseudosymétrie pentamérique autour du canal ionique central (figure 3) [5]. Dans l'organe électrique de la torpille, ce canal est formé de deux sous-unités $\alpha$ identiques qui possèdent chacune un site de liaison de l'ACh, et des sous-unités auxiliaires $\beta$, $\gamma$ et $\delta$.

\section{Organisation structurale des canaux}

Avec leur deux STM par sous unité, KcsA et MscL présentent la topologie minimale pour l'élaboration d'un canal au sein d'une membrane (figures 1 et 2). Dans les deux cas, une hélice délimite la lumière du pore (hélice interne) tandis qu'une autre (l'hélice externe) est localisée à l'extérieur du canal, les parties amino- et carboxy-terminales extramembranaires étant cytoplasmiques. Dans l'homo-tétramère KcsA (figure 1), STMl est en contact avec la membrane lipidique (hélice externe), tandis que STM2 fait face au pore central (hélice interne). (ies deux hélices sont reliées par une trentaine d'acides aminés hautement conservés, qui correspondent au domaine $\mathrm{P}$ crucial pour la conductivité et la sélectivité au $\mathrm{K}^{+}$. Ce domaine se décompose en trois parties fonctionnelles: la tourelle, l'hélice du pore et le filtre de sélectivité constitué par la séquence signature Val-Gly-Tyr-GlyArg. Les quatre hélices internes du pore, à la manière d'un tipi indien inversé, pointent vers le centre de la membrane délimitant une cavité cen- 


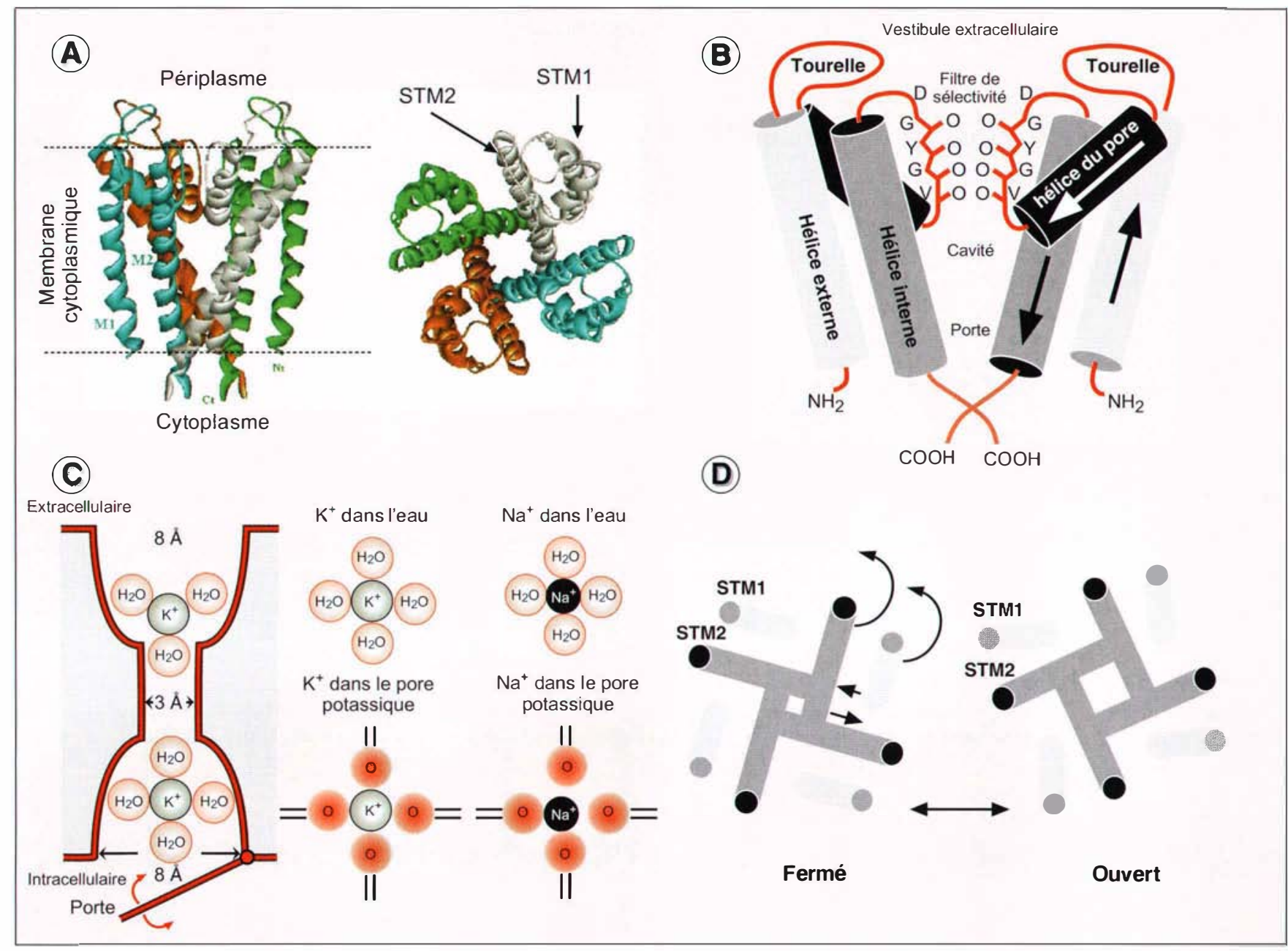

Figure 1. Structure tridimensionnelle du canal potassique KcsA de streptomyces lividans. Cette structure a été obtenue par diffraction de rayons $X$ avec une résolution de 3,2 $\AA$. A. Vue de profil et de dessus du tétramère de sous-unités $\alpha$, chaque sous-unité étant formée de deux segments transmembranaires et d'un domaine $P$. Les sous-unités sont colorées individuellement. B. Structure schématique du pore du canal illustrant les différents domaines fonctionnels. Pour plus de clarté, les sous-unités situées au premier et à l'arrière plans ont été supprimées. Les "O"symbolisent les atomes d'oxygène des liaisons peptidiques de la séquence du filtre de sélectivité (d'après [18]). C. Origine de la sélectivité pour le $K^{+}$au détriment du $\mathrm{Na}^{+}$. La sélectivité dépend de la coordination stricte du $\mathrm{K}^{+}$par les oxygènes des liaisons peptidiques du filtre de sélectivité. Le $\mathrm{Na}^{+}$est plus stable sous la forme hydratée que sous une forme coordonnée du fait de la rigidité de ce filtre. Par conséquent, le $\mathrm{Na}^{+}$reste sous une forme hydratée trop volumineuse pour passer le filtre (d'après [19]). D. Mécanisme moléculaire d'ouverture du canal KCSA, déduit des expériences de RPE. Ce modèle est une vue du côté extracellulaire de la membrane. L'ouverture débute par une étape dépendante du proton. STM2 subit une rotation vers la gauche et s'écarte du centre, permettant ainsi l'ouverture du canal. À la périphérie, STM1 subit la même rotation mais s'incline vers le centre du canal. Les mouvements décrits dans cette illustration sont amplifiés pour plus de clarté (d'après [6]).

trale de $10 \AA$ de diamètre et s'ouvrent à la surface extracellulaire pour présenter le filtre de sélectivité. Ce type d'organisation des hélices internes en cône est un caractère que l'on retrouve dans les deux autres canaux ioniques.

Dans la structure pentamérique du MscL, qui rappelle une bouteille de vin à l'envers (figure 2), c'est le premier seg- constitue l'hélice interne et participe à la formation du pore tandis que STM2 est projeté à l'extérieur du canal. Les cinq hélices internes sont orientées selon un cône et délimitent les parois du pore dont la zone de constriction maximale est riche en acides aminés hydrophobes. Tandis que les hélices externes forment une couronne extéricure autour du pore, les cinq parties carboxy-terminales relativement longues se regroupent selon un faisceau hélicoïdal cytoplasmique.

A partir de l'analyse de sa séquence primaire, il a été prédit que chaque sous-unité constitutive du nAChR comporte un large domaine N-terminal extracellulaire et quatre STM (STM1-4). La carte de densité électronique montre la présence au niveau de la membrane de cinq densités circulaires allongées qui sont typiques 


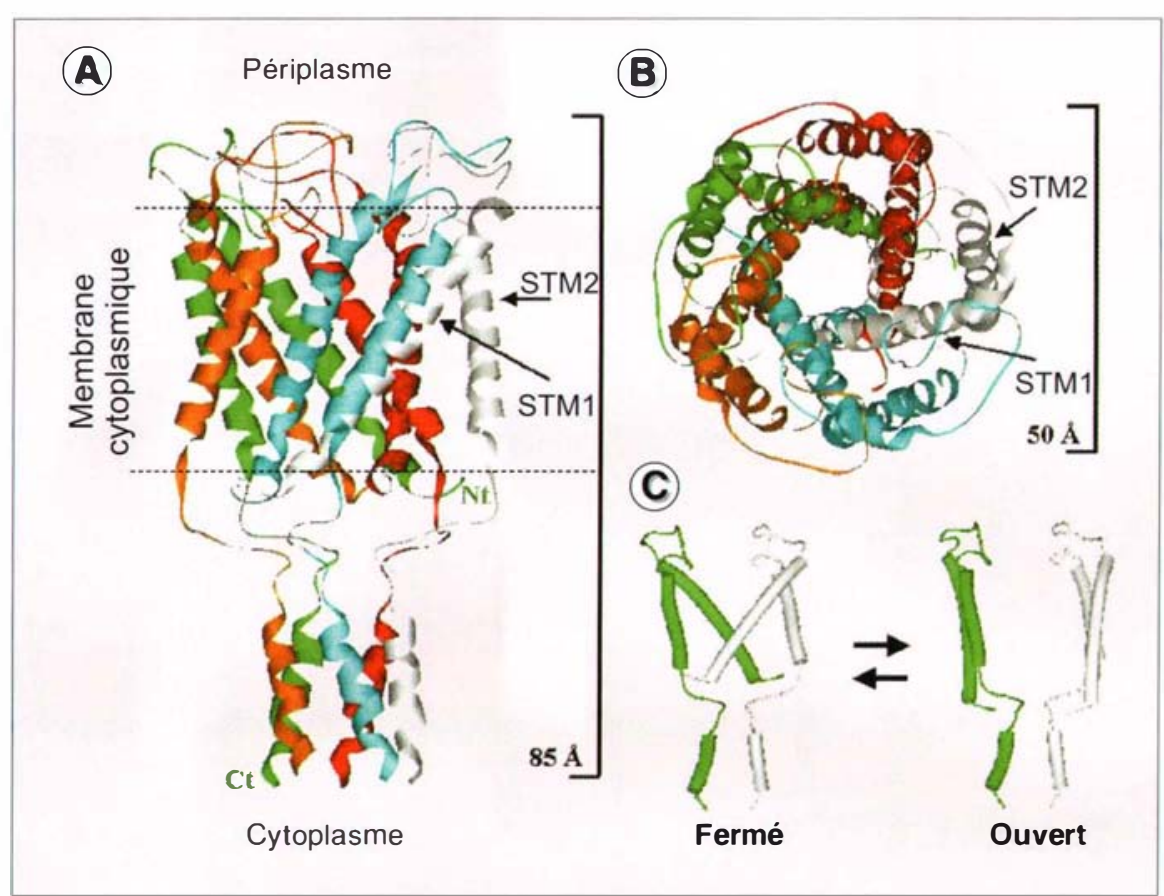

Figure 2. Structure tridimensionnelle du canal mécanosensible MscL de Mycobacterium tuberculosis. Cette structure a été obtenue par diffraction de rayons $X$ avec une résolution de 3,5 $\AA$ (d'après [3]). Vue de profil (A) et de dessus (B) de l'homo-pentamère MscL. Les cinq monomères au sein du canal sont individuellement colorés. Le monomère est constitué de deux segments transmembranaires STM1 et STM2 reliés par une boucle externe et d'une hélice cytoplasmique. Les extrémités $C$ - et $\mathrm{N}$-terminales sont localisées dans le cytoplasme. C. Modèle hypothétique du mécanisme moléculaire d'ouverture du MscL. Par souci de simplicité, deux monomères ont été représentés. Le canal est fermé par les hélices STM1. Une augmentation de la tension membranaire entraînerait un mouvement des hélices et donc une modification des interactions entre les hélices STM1 et STM2. II en résulterait une intercalation des hélices STM2 entre les hélices STM1 à l'origine du pore ouvert.

de structures hélicoïdales formant la paroi du canal ionique central, et qui correspondent aux segments STM2 des cinq sous-unités (figure 3). ('es segments sont séparés de la bicouche lipidique par des structures de densité continue, ce qui suggère que les autres segments hydrophobes traversent la membrane dans une configuration de feuillets $\beta$. L Les segments STM2 sont coudés en leur milieu, et les angles formés pointent vers l'axe du canal, créant ainsi le point de constriction du canal. L'alignement des séquences en acides aminés de ces segments suggère que la constriction résulterait à des interactions entre des résidus hydrophobes (Leu 251) qui formeraient ainsi un anneau central bloquant l'ouverture dlu pore, été abordée de manière indirecte par la technique de résonance paramagnétique électronique (RPE), qui n'exige pas l'obtention de cristaux. En utilisant cette approche, Peromo of al. [6] ont pu mettre en évidence le mouvement des hélices au cours de l'ouverture du KcsA. L orsque le canal est activé, les hélices internes STM2 s'ćcartent et permettent d'augmenter le diamètre de la voic de perméation au niveau du point de convergence des hélices internes (figure 1). Cette région constituerait donc bien la porte du canal.

Un des objectifs majeurs à l'origine des analyses structurales du MscI. était de comprendre les changements de conformation induits par une variation de tension membranaire. Des études de perméabilité ont permis d'estimer la taille du pore à $40 \AA$, ce qui rend compte de la grande conductance du canal [11]. Pour créer un tel pore, un large mouvement des hélices apparaît nécessaire et conduirait à l'intercalation des hélices externes et internes. En accord avec cette hypothèse, des expériences de protéolyse et de mutagenèses dirigée impliquent à la fois des régions membranaires et extramembranaires dans la transduction du signal mécanique $[12,13]$.

Un mouvement des hélices a été également décrit dans le cas du nAChR [14]. Dans la configuration ouverte du canal, visualisée par congélation rapide après la vaporisation d'ACh, la courbure des segments STM2 n'apparaît plus dirigée vers le centre du pore, mais orientée de côté. La liaison de l'A('h induit donc la rotation des hélices, dont la courbure devient alors tangentielle au canal (figure 3). ('eci permettrait d'exposer les acides aminés polaires vers la lumière du canal et d'enlever les chaines latérales encombrantes des résidus Leu du centre du pore, ouvrant ainsi le passage aux ions.

L.es structures tridimensionnelles précédemment décrites concernent les canaux dans leur état fermé. Une connaissance précise du mécanisme moléculaire de fonctionnement des canaux ioniques nécessiterait la détermination de leur structure à l'état ouvert. Compte tenu de la difficulté d'obtenir des cristaux de canaux figés dans leur état ouvert, cette question a

\section{Que nous apprend la structure} sur le mécanisme de la sélectivité des canaux ?

Ces informations structurales ont permis, dans le cas du KcsA, d'élucider les mécanismes à l'origine de sa haute sélectivité (perméabilité 10000 fois plus grande au $\mathrm{K}^{+}$qu'au $\mathrm{Na}^{+}$) et de sa 


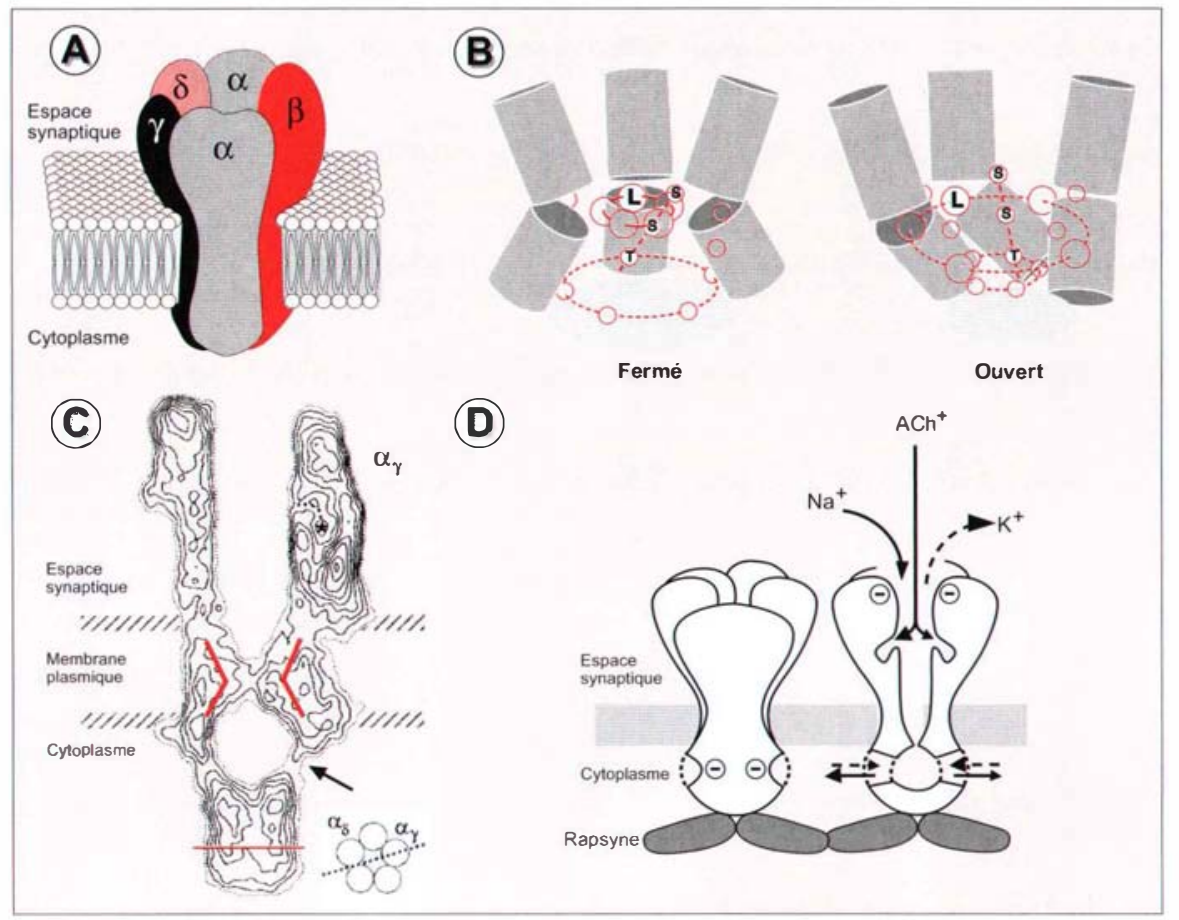

Figure 3. Structure tridimensionnelle du récepteur nicotinique. Cette structure a été obtenue par diffraction électronique de cristaux bidimensionnels à $4,6 \AA$ de résolution. A. Schéma montrant la stœchiométrie pentamérique du récepteur nicotinique. B. Représentation en densité électronique de la section longitudinale du récepteur passant par l'une des sous-unités $\alpha$ (voir insert). L'astérisque indique la cavité dans la partie extracellulaire de la sousunité $\alpha$ qui constituerait le site de liaison de l'ACh, cavité reliée au vestibule par un étroit tunnel (ligne pointillée). La flèche indique une ouverture dans la partie cytoplasmique du récepteur. La structure située sous la ligne continue est constituée par une protéine associée, la rapsyne (d'après [5]). C. Modèle du mécanisme d'ouverture du canal: le changement de conformation des hélices permettrait de dégager les chaînes latérales des résidus Leu localisés à l'angle de la courbure des hélices, et d'exposer les chaînes d'acides aminés polaires, ouvrant le pore au passage des ions (d'après [14]). D. Modèle de perméation des ions à travers le canal. Le filtre de sélectivité serait assuré par les charges négatives situées dans le vestibule externe et aux abords des ouvertures dans la partie cytoplasmique du récepteur (d'après [5]).

rapidité de conduction (chaque canal permet le passage de $10^{8}$ ions $\mathrm{K}^{+}$par seconde). Les régions extracellulaires et intracellulaires du pore sont chargées négativement grâce aux chaînes latérales d'acides aminés. Ceci a pour conséquence d'augmenter la concentration relative locale en cations. En partant de la face intracellulaire du canal, le pore est constitué d'une partic interne de $18 \AA$ de long qui s'ouvre ensuite sur la cavité centrale (figure 1). Dans ces deux compartiments dont les parois sont hydrophobes, les ions $\mathrm{K}^{+}$ sont hydratés. En revanche, le filtre de sélectivité qui sépare la cavité et le

(environ $3 \AA$ ) que les ions $\mathrm{K}^{+}$ne pourront passer que sous une forme déshydratée. Cé filtre, délimité par les atomes d'oxygène des liaisons peptidiques de la séquence signature du domaine $\mathrm{P}$, est très polaire. Au niveau électrostatique, un ion $\mathrm{K}^{+}$doit franchir une barrière énergétique maximale au centre de la membrane. La cavité centrale aqueuse constitue un milieu polarisé qui évite la déstabilisation électrostatique de l'ion $\mathrm{K}^{+}$. De plus, les quatre hélices du pore pointent vers le centre de la cavité et imposent un potentiel électrostatique négatif [15]. Ces deux facteurs abaissent la barrière électrostatique, facilitant ainsi le pas- sage des ions $\mathrm{K}^{+}$. L'aspect hydrophobe des parois de la cavité centrale favorise le flux rapide de $\mathrm{K}^{+}$au travers du pore car les ions interagissent uniquement avec les molécules d'eau et avec le filtre de sélectivité. La structure du filtre de sélectivité présente deux caractéristiques. D'une part, les atomes d'oxygène des liaisons peptidiques de la séquence signature forment des anneaux séquentiels empilés qui définissent plusieurs sites très rapprochés et qui permettent la diffusion rapide des ions $\mathrm{K}^{+}$déshydratés (figure 1). D'autre part, les chaînes latérales de la Val et de la Tyr de la séquence signature pointent vers l'extérieur du pore et établissent des liaisons spécifiques avec certains acides aminés des hélices du pore, imposant une contrainte physique sur la taille de ce demier. Cette structure rend compte de la prodigieuse sélectivité du pore. Quand un ion pénètre dans le filtre, il va se trouver sous une forme déshydratée. Pour compenser le coût énergétique de la déshydratation, les atomes d'oxygène des liaisons peptidiques vont mimer ceux des molécules d'eau (figure 1). La structure révèle que le filtre de sélectivité ne pourra pas coordonner convenablement un ion de plus petit diamètre comme le sodium. La compensation du coût énergétique de la déshydratation de l'ion $\mathrm{Na}^{+}$ne sera donc pas possible, et il restera sous unc forme hydratée trop volumineuse pour passer. Le filtre de sélectivité peut accommoder deux ions $\mathrm{K}^{+}$déshydratés espacés de $8 \AA$, dont la présence simultanée induit une répulsion mutuelle qui contribue à la conduction. Ce mécanisme rend compte à la fois de la forte interaction de l'ion avec le filtre de sélectivité et du flux très important induit par la répulsion électrostatique des ions.

Sans atteindre la finesse de l'analyse précédente, deux parties extramembranaires ont été impliquées dans le mécanisme de sélectivité du nAChR, un canal cationique perméable à la fois aux ions $\mathrm{Na}^{+}$et $\mathrm{K}^{+}$. On a identifié, dans le vestibule extracellulaire et audessus des poches représentant très probablement les sites de fixation pour l'ACh, des acides aminés chargés négativement qui seraient responsables du filtre de sélectivité externe. 
Par ailleurs, la paroi cytoplasmique du récepteur, formé principalement par la boucle reliant STM3 et STM4, et qui délimite la cavité interne du canal, contient des ouvertures relatirement étroites $(<10 \AA)$. ('elles-ci serviraient de filtre excluant de la bouche cytoplasmique du canal les anions (par la charge négative des chaînes latérales d'acides aminés), ainsi que les gros cations et les molécules non perméantes.

\section{Conclusions}

Toutes ces nouvelles données suggèrent qu'il existe une similitude architecturale de ces canaux: les segments hélicoïdaux sont organisés en cônes autour du canal central et convergent en un point de constriction constituant la porte du canal. Une rotation de ces segments, augmentant le diamètre du pore au niveau du point de constriction, serait à l'origine de l'ouverture du pore. La sélectivité ionique résulterait à la fois des charges des acides aminćs présents dans les vestibules internes et externes du pore et, dans le cas du KcsA, d'une filtration par la taille des ions déshydratés. Cependant, si les bases moléculaires de la sélectivité ionique et des mécanismes à l'origine de l'ouverture du pore se précisent, il reste encore à établir comment les canaux répondent aux stimulus responsables du changement de conformation entre états fermé et ouvert.

Ces informations structurales viennent heureusement couronner un siècle cl'études électrophysiologiques sur les transferts d'ions à travers les membranes. L'irruption des bactéries dans le domaine n'est pas fortuite: elle s'explique par le fait que les études cristallographiques requièrent une masse importante de protéines. Deux autres canaux bactériens ont été mis en évidence: un canal chlorure de la famille des CIC [16] et un canal activé par le glutamate [17]. Nul doute que leur cristallisation sera tentée. On peut légitimement supposer que ces structures de canaux "primitifs" possèdent des caractères communs avec ceux des canaux eucaryotes. Dans cette perspective, les études de biologie structurale vont certainement jouer un $m / s n^{\circ}$ G-i. vol. Iis, juin-juillet 2001 rôle déterminant pour la compréhension du fonctionnement (et du dysfonctionnement) des canaux ioniques des animaux supérieurs

\section{Remerciements}

Nous remercions Alexandre Ghazi pour sa lecture critique du manuscrit et Kyu Park et Mireille Passama pour leur aide technique clans l'élaboration des figures.

\section{Catherine Berrier}

Groupe des canaux ioniques, Cnrs UMR 8619, Université Paris-Sud, Bât. 430, 91405 Orsay Cedex, France.

\section{Éric Honoré}

IPMC-Cnrs, 660, route des Lucioles Sophia Antipolis, 06560 Valbonne, France.

\section{Isabel A. Lefèvre}

Neurophysiologie, Sanofi-Synthélabo, 371, rue du Pr-J.-Blayac, 34184 Montpellier Cedex, France.

\section{Nicolas Hussy}

Biologie des Neurones endocrines, Cnrs UMR 5101, 141, rue de la Cardonille, 34094 Montpellier, France.

Les auteurs font partie du comité d'organisation du Colloque Canaux Ioniques. Site web: http://physio.univ-lyon 1.fr/ canaux/canaux.htm

\section{RÉFÉRENCES}

1. Schrempf H, Schimdt O, Kummerlen R, et al. A prokarietic potassium channel with two predicted transmembrane segments from Streptomyces lividans. EMBO J 1995; 14: 5170-8.

2. Doyle DA, Cabral JM, Pfuetzner RA, et al. The structure of the potassium channel: molecular basis of potassium conduction and selectivity. Science 1998; $280: 69-77$.

3. Chang G, Spencer RH, Lee AT, Batclay M, Rees D. Structure of the MscL homolog from Mycobacterium tuberculosis: a gated mechanosensitive channel. Science 1998; 282: 2220-5.

4. Unwin N. Nicotinic acetylcholine receptor at $9 \AA$ resolution. J Mol Biol 1993; 229. tor at $1101-24$

5. Miyazawa A, Fujiyoshi Y, Stowell M, Unwin N. Nicotinic acetylcholine receptor at $4.6 \AA$ resolution: transverse tunnels in the channel wall. J Mol Biol 1999; 288: 765-86.
6. Perozo E, Cortes DM, Cuello LG. Structural rearrangements underlying $\mathrm{K}^{+}-$channel activation gating. Science 1999 ; 285 : 73-8.

7. Sukharev SI, Blount P, Martinac B, Kung C. A large conductance mechanosensitive channel in $E$. coli encoded by msd alone. Nature 1994; 368: 256-68.

8. Berrier C, Coulombe A, Szabo I, Zoratti M, Ghazi A. Gadolinium ion inhibits loss of metabolites induced by osmotic downshock, and large stretch activated channels in bacteria. Eur J Biochem 1992 ; 206 : 559-65.

9. Levina N, Tötemeyer S, Stockes NR, Louis P, Jones MA, Booth IR. Protection of $E$. coli cells against extreme turgor by activation of MscS and MscL mechanosensitive channels: identification of genes required for MscS activity. EMBO J 1999; 18: 1730-7.

10. Revah F, Bertrand D, Galzi JL, et al. Mutations in the channel domain alter desensitization of a neuronal nicotinic receptor. Nature 1991 ; 353: 846-9.

11. Gruickshank C, Minchin RF, LeDain AC, Martinac B. Estimation of the pore size of the large conductance mechanosensitive ion channel of Escherichia coli. Biophys $J$ 1997; 73: 1925-31.

12. Yoshimura K, Batiza A, Schroeder M, Bount $P$, Kung C. Hydrophilicity of a single residue within $\mathrm{MscL}$ correlates with increases channel mechanosensitivity. Biophys J 1999; 77 : 1960-72.

13. Ajouz B, Berrier C, Besnard M, Martinac B, Ghazi A. Contributions of the different extra-membranous domains of the mechanosensitive ion channel MscL to its reponse to membrane tension. J Biol Chem 2000; 275: 1015-22.

14. Unwin N. Acetylcholine receptor channel imaged in the open state. Nature 1995; $373: 37-43$.

15. Roux B, Mackinnon R. The cavity and pore helices in the $\mathrm{KcsA} \mathrm{K} \mathrm{K}^{+}$channel: electrostatic stabilization of monovalent cations. Science 1999 ; 285 : 100-2.

16. Maduke M, Pheasant DJ, Miller C. High level espression, fonctional reconstitution, and quaternary structure of a prokaryotic CIC-type chloride channel. J Gen Physiol 1999; 114: 713-32.

17. Chen GQ, Cui C, Mayer ML, Gouaux E. Functional characterization of a potassiumselective prokaryotic glutamate receptor. Nature 1999; 402: 817-21.

18. Choe S, Robinson R. An ingenious filter: the structural basis for ion channel selectivity. Neuron $1998 ; 20: 821-3$.

19. Armstrong $C$. The vision of the pore. Science $1998 ; 280: 56-7$.

\section{TIRÉS À PART}

(.. Berrier. 